\title{
The role of cell type-specific mitochondrial dysfunction in the pathogenesis of Alzheimer's disease
}

\author{
Dong Kyu Kim \& Inhee Mook-Jung ${ }^{*}$ \\ Department of Biomedical Sciences, College of Medicine, Seoul National University, Seoul 03080, Korea
}

\begin{abstract}
The decrease of metabolism in the brain has been observed as the important lesions of Alzheimer's disease (AD) from the early stages of diagnosis. The cumulative evidence has reported that the failure of mitochondria, an organelle involved in diverse biological processes as well as energy production, maybe the cause or effect of the pathogenesis of AD. Both amyloid and tau pathologies have an impact upon mitochondria through physical interaction or indirect signaling pathways, resulting in the disruption of mitochondrial function and dynamics which can trigger AD. In addition, mitochondria are involved in different biological processes depending on the specific functions of each cell type in the brain. Thus, it is necessary to understand mitochondrial dysfunction as part of the pathological phenotypes of $A D$ according to each cell type. In this review, we summarize that 1) the effects of AD pathology inducing mitochondrial dysfunction and 2) the contribution of mitochondrial dysfunction in each cell type to AD pathogenesis. [BMB Reports 2019; 52(12): 679-688]
\end{abstract}

\section{INTRODUCTION}

Alzheimer's disease (AD) accompanied by extracellular amyloid plaques and intracellular neurofibrillary tangles exhibits memory impairment and cognitive deficit in patients with $A D$ (1). However, the underlying mechanisms of the pathogenesis of $A D$ remain unclear, and therapeutic approaches directly targeting amyloid beta $(A \beta)$ and tau have failed $(2,3)$. The development of ${ }^{18} \mathrm{~F}$-Fluorodeoxyglucose positron emission tomography (FDG-PET) which visualizes the usage of glucose in the tissue, reveals the association between reduced metabolism in the brain and $A D$ pathogenesis (4). In the progress of $A D$, since metabolic defect of the brain has appeared as the early symptoms of $A D$ even before onset of

*Corresponding author. Tel: +82-2-3668-7636; Fax: +82-2-3672-7352; E-mail: inhee@snu.ac.kr

https://doi.org/10.5483/BMBRep.2019.52.12.282

Received 4 November 2019

Keywords: Alzheimer's Disease, Bioenergetic Defects, Mitochondria, Mitochondrial Dynamics, Mitochondrial Dysfunction
AD pathological symptoms with brain atrophy and memory loss, the reduction of FDG-PET has long been used for the imaging biomarker of $\mathrm{AD}$ (5). Hypometabolism in the brain of $A D$ is attributed to abnormal morphology and impaired functions of mitochondria $(6,7)$. For this reason it is noted that mitochondria are responsible for energy supply and maintenance of different functions of cells and mitochondrial failure has been reported in patients with $A D(8)$. It has been suggested that mitochondrial dysfunction and impaired dynamics appear to be critical roles in the pathogenesis of $A D$ $(9,10)$. The mitochondrial cascade hypothesis has been postulated to explain the onset of bioenergetics dysfunction involved in the pathogenesis of $A D(11,12)$. The hypothesis assumes that gene inheritance and environmental factors regulate mitochondrial functions, which in turn determines the vulnerability to $A D(13)$. Also, Both amyloid and tau pathology can induce mitochondrial alterations in vitro and vivo, indicating that bioenergetics dysfunction is closely associated with $A D$ pathology $(14,15)$. In this review, we discuss the mitochondrial failure affected by $\mathrm{AD}$ pathology, and its implication in different cell types for the pathogenesis of AD.

\section{MITOCHONDRIAL DYSFUNCTION INDUCED BY ALZHEIMER'S DISEASE PATHOGENESIS}

\section{Mitochondrial bioenergetics defects}

The metabolism and glucose uptake of the brain tissue is down-regulated in patients with $A D(16,17)$. The investigation of bioenergetics profiles of fibroblasts from late-onset $A D$ (LOAD) and health control demonstrates that the cells from LOAD, have the metabolic shift from the mitochondrial oxidative phosphorylation system (OXPHOS) to glycolysis, indicating reduced mitochondrial metabolic potential in LOAD (18). Mitochondria fractioned from triple transgenic AD model mice (3xTg-AD) brains show a decrease in mitochondrial membrane potential, ATP/ADP ratio and an impairment of the respiratory activities (19). The brain tissue of APP/PS1 AD model mice contains fewer ATP contents compared to the wild-type mice brain sample from 5 months old (20). When $A \beta$ is specifically accumulated in mitochondria by using mitochondria-targeted $A \beta$ construct, various mitochondrial functions were impaired, including the mitochondrial membrane potential and ATP generation (21) 


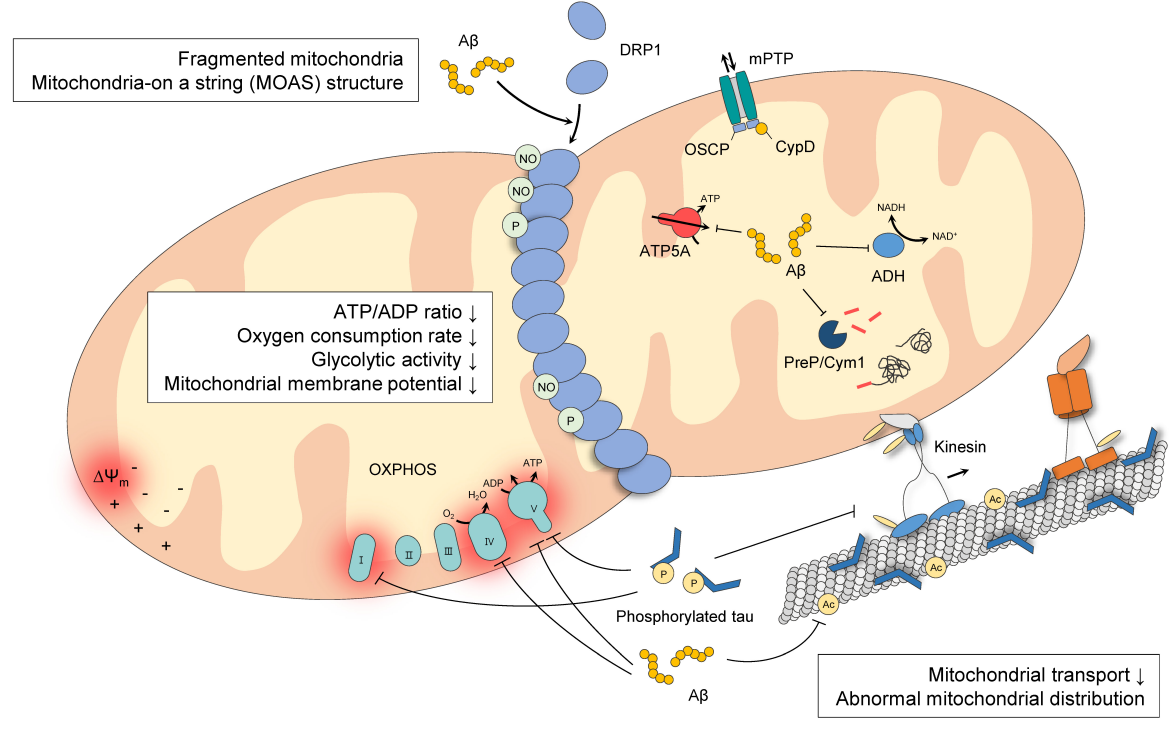

Fig. 1. Mitochondrial alterations in AD. The effect of $A D$ pathology on mitochondrial function for energy production, transport and dynamics.
(Fig. 1). The genetic isoforms of apolipoprotein $E$ (ApoE), the leading risk factor for the onset of LOAD, are also known to affect cellular metabolism (22). When each ApoE isoform is overexpressed in the mouse neuroblastoma cell line, the levels of hexokinase, one of the glycolytic enzymes, and the glycolytic activity are reduced in ApoE4-overexpressing cells as compared to other isoforms. In addition, it is shown that the oxygen consumption rate and the ATP amounts produced through the OXPHOS system are also shown to decrease when ApoE4 is overexpressed (23).

Many previous studies have investigated that there are distinct pathways how $A \beta$ affects mitochondrial respiratory complexes. Both overexpression of amyloid precursor protein (APP) in cells and transgenic AD model mice represent reduced activities of adenosine $5^{\prime}$-triphosphate synthase (ATP synthase, mitochondrial complex V), but not other complexes, leading to reducing oxygen consumption and ATP production $(24,25)$. Using proteomic and functional analysis, differentially expressed proteins in $\mathrm{P} 301 \mathrm{~L}$ tau transgenic mice brain are identified as compared to wild-type mice brain, which are involved in a metabolism and mitochondrial respiration process (26). A decrease in complex I activity and ATP synthesis is observed in P301L mice brain. In addition, human FTDP-17 patients with P301L tau mutation show reduced complex $\mathrm{V}$ levels in the cortex region of the brain. Here it is noted that the SH-SY5Y cell line overexpressing human P301L mutant tau exhibits decreased complex I activity, as accompanied by decreased ATP levels (27). When 3xTg-AD mouse model with both amyloid and tau pathology is compared with $\mathrm{AD}$ mouse model with distinct single pathology, the synergistic effects of both pathologies are the impact on the OXPHOS. In consistent with previous reports, mitochondrial complex I is down-regulated dependent of tau pathology whereas complex IV is affected by amyloid pathology at protein and activity levels (28). Together, each amyloid and tau pathology impact individually on the functions of mitochondrial components, and both pathologies synergistically induce mitochondrial failure in AD (Fig. 1).

\section{Interaction of $\mathbf{A} \beta$ with mitochondrial components}

It has been reported that $A \beta$ is accumulated within the mitochondria of $\mathrm{AD}$ brain tissue $(29,30)$. $A \beta$ can be translocated into mitochondrial matrix via the import machinery of mitochondria and APP is embedded in mitochondrial membrane, resulting in causing mitochondrial toxicity $(21,31$, 32). A $\beta$ located to the mitochondrial matrix can physically interact with mitochondrial components, thereby inhibiting their functions and producing excessive oxidative stress (Fig. 1) $(33,34)$. ATP synthase is localized in the inner membrane of mitochondria as the last component of the electron transport chain, where it produces ATP by the flux of a proton gradient across mitochondrial inner membrane (35). It has been reported that $A \beta$ binds to ATP synthase and dysregulates its function, thereby inhibiting energy production. ATP synthase subunit $\alpha$ (ATP5A) activity which is regulated by the attachment of O-linked $\mathrm{N}$-acetylglucosamine (O-GlcNAcylation) can be inhibited by the binding of A $\beta$ to ATP5A. A $\beta$ disrupts the interaction between ATP5A and O-GlcNAc transferase, resulting in blocking O-GlcNAcylation of ATP5A (36).

One of possible mechanisms to induce neuronal toxicity by $A \beta$ is to form the mitochondrial permeability transition pore (mPTP), which activates the apoptotic pathway by the efflux of $\mathrm{Ca}^{2+}$ and apoptotic factors from the mitochondrial matrix (37, 38). Cyclophilin D, a peptidylprolyl isomerase $F$, is known to regulate the opening of $\mathrm{mPTP}$ pore in the mitochondrial matrix $(39,40)$. The physical interaction of cyclophilin $D$ with $A \beta$ 
occurs in the mitochondrial matrix, resulting in the inhibition of cyclophilin D to close mPTP pore. The pathological features of $A D$ including mitochondrial toxicity and neuronal dysfunction can be reduced by genetic deletion of cyclophilin $D$, indicating that the cyclophilin $D-A \beta$ interaction resulting in mPTP opening promotes $A \beta$-induced pathology of AD (41). Also, oligomycin sensitivity conferring protein (OSCP) subunit of ATP synthase involved in the formation of mPTP with cyclophilin $D$, also has the physical interaction with $A \beta$. The interaction leads to disrupting the stability and activity of ATP synthase, increased oxidative stress, and activated mPTP but the activities of other OXPHOS complexes are noted to be relatiely unchanged (25).

Alcohol dehydrogenase, which catalyzes the reduction of the nicotinamide adenine dinucleotide $\left(\mathrm{NAD}^{+}\right)$to $\mathrm{NADH}$ using alcohol, is suppressed by $A \beta$ in the mitochondrial matrix of AD patients and transgenic model mice (42). In these cases, $A \beta$ induces to deform the active site of alcohol dehydrogenase, resulting in the inhibition of $\mathrm{NAD}^{+}$binding. The mouse model in which alcohol dehydrogenase is overexpressed in an $A \beta$-rich environment exhibits a memory deficit dependent of the hippocampus, indicating that $A \beta$-induced mitochondrial toxicity occurs through the interaction between alcohol dehydrogenase and $A \beta$.

Mitochondrial proteins encoded by nucleus DNA possess the signal peptide to pull it into the mitochondrial matrix. After the import, the mitochondria-targeting sequence is cleaved by the mitochondrial processing peptidase (43). In the mitochondrial matrix, peptidasome Cym1/PreP degrades presequence peptides of mitochondrial proteins. $A \beta$ accumulated in mitochondria can disrupt PreP, thereby inhibiting the cleavage of presequence peptides. Consequently, an accumulation of undegraded presequence peptides cause feedback inhibition of preprotein processing. Damaged mitochondrial protein maturation induces mitochondrial toxicity and alteration of the mitochondrial proteome in AD patients (44).

\section{Mitochondrial dynamics and homeostasis}

Since mitochondrial morphology and dynamics are closely associated with mitochondrial functions and their homeostatic maintenance, it is shown that mitochondria respond to energetic demands through a process of fusion/fission dynamics $(45,46)$. Using an electron microscopy, an abnormal mitochondrial morphology is observed in the brain of $A D$ (47-49). The long connected mitochondria termed mitochondria-on a string (MOAS) as a result of fission arrest, are observed in the hippocampus and entorhinal cortex of $A D$ patients and $A D$ model $(47,50,51)$. In $A D$ model mice (APPSwe:PSEN1 $\triangle E 9$ ), mitochondrial loss and abnormal structure of mitochondria, particularly mitochondrial swelling, are observed near amyloid plaques. The neurons affected by near amyloid plaques contain highly fragmented mitochondria as compared to distinct neurons from amyloid plaques and neurons of wild-type mice (52). In addition, fibroblasts obtained from $A D$ patients presents a decrease in the mitochondrial length (53).

With morphological changes of mitochondria, the machinery required for mitochondrial dynamics, such as mitochondrial fusion proteins (OPA1, MFN1, and MFN2), is altered in the hippocampus of $\mathrm{AD}$ brain, seemingly without any change of the total levels of mitochondrial components (45). The activity of dynamin-related protein1 (DRP1), one of key regulators for mitochondrial fission, is elevated in the brain of subjects with $\mathrm{AD}$, which can translocate to mitochondrial outer membrane and then leads to mitochondrial fission, but mitochondrial fusion proteins, such as MFN1, MFN2 and OPA1, are decreased in AD patients (54). The pharmacological inhibition of DRP1 can restore mitochondrial homeostasis and functions, including membrane potential, ATP production and reactive oxygen species production, and attenuates memory impairment in $\mathrm{AD}$ model mice $(55,56)$. Overexpression of APP and A $\beta$ can affect the mitochondrial dynamics and homeostasis. APP-overexpressing cells exhibit fragmented mitochondria and altered mitochondrial distribution around the nucleus. The levels of DRP1 and OPA1 are decreased, but it is noted that the levels of FIS1 (mitochondrial fission 1 protein) are increased in APP-overexpressing cells (57). Furthermore, DRP1 oligomerization and recruitment on mitochondrial membrane are regulated by its posttranslational modification including phosphorylation of S-nitrosylation (58, 59). A $\beta$ causes nitrosative stress to the cell which promotes S-nitrosylation modification on DRP1, leading to an increase in fission activity and further mitochondrial fragmentation (Fig. 1) $(60,61)$. Increased DRP1 activity due to abnormal interaction with phosphorylated tau can elucidate excessive mitochondrial fragmentation (62). In this case, the genetic reduction of DRP1 protect the mitochondrial dysfunction and impaired dynamics in P301L tau transgenic mice (63). In addition, truncated tau causes mitochondrial fission and a reduction of OPA1 levels in neurons, as compared to wild full-length tau, indicating that different forms of tau have a distinct impact on the mitochondrial dynamics (64). Additionally, CR6-interacting factor 1 (Crif1) involved in both the translation of OXHPHOS proteins and their insertions into the mitochondrial inner membrane is down-regulated by $\mathrm{A} \beta$-induced reactive oxygen species (ROS). As a result, a decrease of Crif1 results in fragmentation, dysfunction of mitochondria and even cell death in the subject with AD (65).

Extensive neurites of neuron require a wide coverage of energy and material supply to maintain neuronal functions. In fact, to deliver the mitochondria to nerve terminals, the neuron uses a microtubule axonal transport system, which can be regulated diverse post-translational modifications, including phosphorylation and acetylation. The levels of acetylated $\alpha$-tubulin are decreased in AD patient's brains and in the hippocampal neurons which are treated with $A \beta$. The inhibition of histone deacetylase 6 which deacetylases $\alpha$-tubulin rescues the inhibited mitochondrial axonal transport by $A \beta$ (Fig. 1) 
(66). The patterns of mitochondrial distribution in hippocampal neurons are seen to be different in AD. Although mitochondria localize at both neuronal process and soma in control group, most mitochondria are confined to the soma area in AD (49). Since tau serves as microtubule-associated protein to stabilize microtubule, tau pathology is therefore associated with an abnormal mitochondrial transport in AD. The overexpression of phosphorylated tau disrupts mitochondrial movement by regulating microtubule spacing (67). In other words, the mitochondrial distribution is altered in neurons with pathological tau aggregates of rTg4510 tau transgenic mice and $\mathrm{AD}$ patients. To this end, a reduction of soluble tau expression can restore the mitochondrial distribution, despite an existence of fibrillary tau inclusions (68). In addition to destabilizing microtubule network, tau also interact with kinesin motor protein, leading to preferential inhibition of anterograde transport along microtubules (Fig. 1) (69). These evidences suggest that amyloid and tau pathology affect mitochondrial dynamics to induce fragmentation and influence microtubule-based transport.

\section{THE EFFECT OF MITOCHONDRIAL DYSFUNCTION ON EACH CELL TYPE IN ALZHEIMER'S DISEASE}

Different cell types in the brain have distinct characteristics of metabolism, and exhibit specific roles related to their metabolic characteristics. Increasing evidences indicate that the mitochondria in different cell types vary in their function and morphology. Recently, the mitochondrial proteome of three major cerebellar cell types is identified, and it suggests that each cell type has differentially regulated mitochondrial proteins based on each biological role as utilized in the brain (70). In general, the metabolic coupling between neuron and astrocyte using mitochondria in different ways, manages and supports the functionality of the brain. In this case, the toxic fatty acids produced from hyperactive neurons are transferred into neighboring astrocytes, which can be stored in lipid droplets or detoxified by the $\beta$-oxidation process in mitochondria rather than processed in the neurons (71). Microglia undergo the metabolic reprogramming mediated by mitochondrial dynamics in response to external stimuli, which determine the inflammatory characteristics of microglia $(72,73)$. A better understanding of mitochondrial dysfunction as a pathological feature of $A D$ requires a cell-type specific approach. We review mitochondrial dysfunction of each cell type, and note their contribution to AD pathogenesis.

\section{Neuron}

Neuron has different compartments with differentially functional units including axon and dendrite. The synaptic functions to release neurotransmitters and to respond signals at postsynaptic region require a high number of mitochondria, because of the high energy demand at the synapses (74). For this reason, the neuron has a high metabolic rate and the supply of glucose determines its functionality in the brain. The synaptic mitochondria especially have discrete metabolic characteristics that they are susceptible to the inhibition of complex I and $\mathrm{Ca}^{2+}$ overload compared to non-synaptic mitochondria $(75,76)$. Since it is noted that the tau pathology has adverse effect upon mitochondrial complex $I$ and $A \beta$ activates synaptic terminals by the influx of $\mathrm{Ca}^{2+}$ into cytosol, it seems likely that the synaptic mitochondria are impaired in $A D(27,28,77)$. The existence of $A \beta$ in synaptic mitochondria has been reported by the immunogold electron microscope (78). Moreover, the synaptic mitochondria contain higher amounts of $A \beta$ as compared to non-synaptic mitochondria in Tg mAPP AD model mice, resulting in the impairment of synaptic mitochondrial respiration and accumulation of oxidative stress at synapses (Fig. 2) (78). The AD patient brain has local differences in the number of synaptic mitochondria as well as functional abnormality. For example, it is seen that the presynaptic region in $A D$ has fewer mitochondria with abnormal morphology and structure, as compared to control subject, but there is no difference in those of a comparison post-synaptic region (79).

The synaptic communication between neurons is regulated by $\mathrm{Ca}^{2+}$ signaling through the binding of neurotransmitters and their receptors at post-synaptic region. In fact, the synaptic mitochondria damaged by oxidative stress or $A D$ pathology lose the capability to buffer excessive cytosolic $\mathrm{Ca}^{2+}$ concentration. The expression of mitochondrial $\mathrm{Ca}^{2+}$ exchange transporter $\mathrm{NCLX}, \mathrm{Na}^{2+} / \mathrm{Ca}^{2+}$ exchanger, is decreased in the brain of AD patients and 3xTg-AD model mice. Furthermore, the genetic deletion of NCLX leading to impaired mitochondrial $\mathrm{Ca}^{2+}$ efflux can cause memory loss, and aggravate both amyloid and tau pathology. Restoration of mitochondrial exchange transporter in neurons rescues mitochondrial dysfunction, cognitive impairment and AD pathology (Fig. 2) (80). $\mathrm{Ca}^{2+}$ dysregulation of presynaptic mitochondria in mossy fiber synapses is exhibited in Tg2576 AD model mice. Moreover, it is shown that an exposure of $A \beta$ to granule cells of the dentate gyrus causes $\mathrm{Ca}^{2+}$ clearance failure. The results support that mitochondrial dysfunction by overproduced or existence of $\mathrm{A} \beta$, particularly mitochondrial $\mathrm{Ca}^{2+}$ regulation, is implicated in the synaptic dysfunction of mossy fiber-CA3 synapses (81). Similarly, impaired long-term potentiation and short-term plasticity at the mossy fiber synapses in Presenilin knockout mice are resulted from the altered mitochondrial $\mathrm{Ca}^{2+}$ homeostasis in granule cells (82). The insulin-like growth factor-1 (IGF-1) signaling increased in AD patients and AD model mice is regulated by mitochondrial $\mathrm{Ca}^{2+}$ homeostasis, which activates to release neurotransmitters and basal synaptic transmission (83-85). The pharmacological blockade of IGF-1 signaling can attenuate hippocampal hyperactivity in APP/PS1 model mice, indicating that mitochondrial dysfunction in $A D$ conditions fails to control $A \beta$-dependent neuronal activation which is caused by excessive IGF-1 signaling (Fig. 2) (83). 


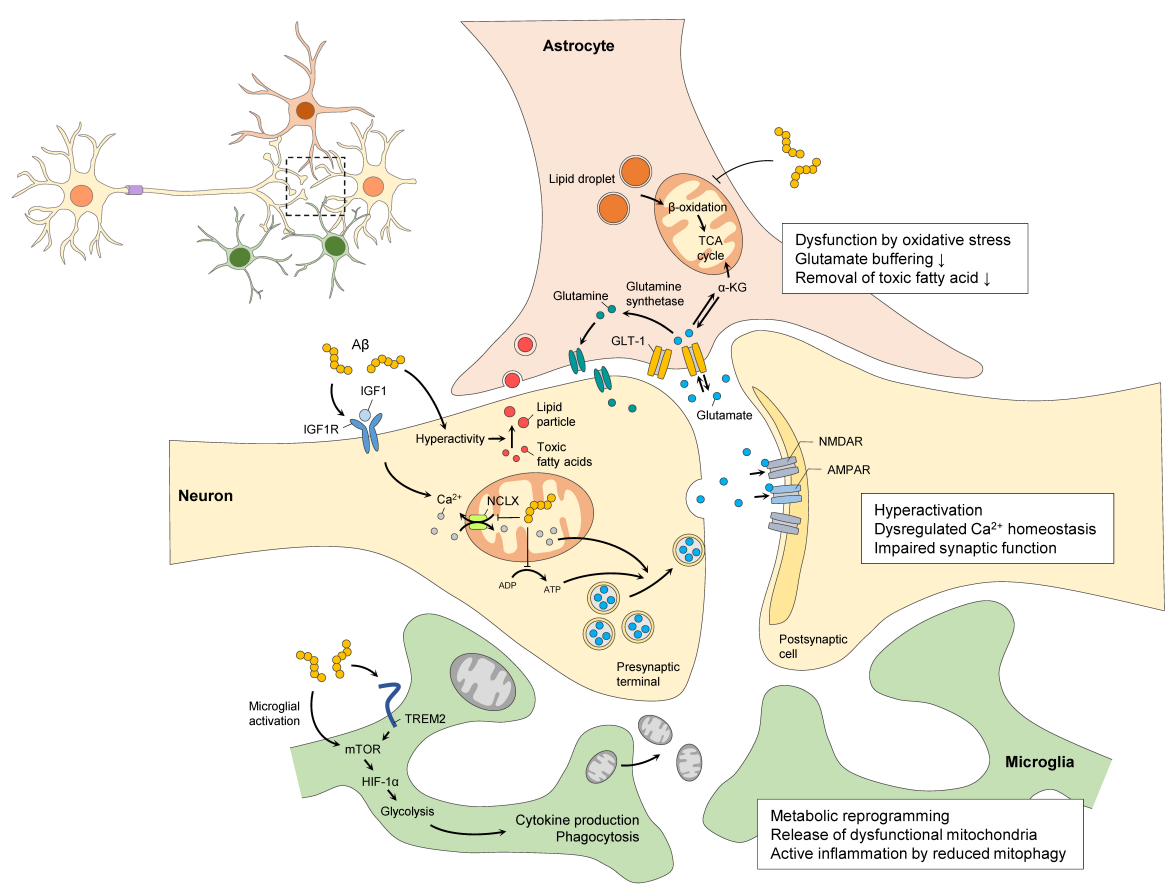

Fig. 2. Cell type-specific mitochondrial dysfunction in $\mathrm{AD}$ pathogenesis. Many mitochondria are located in nerve terminals, contributing to supply energy for the production of neurotransmitters and the transport and release of synaptic vesicles. The damage of synaptic mitochondria causes abnormal synaptic activity in AD. Astrocyte regulates neuronal activity by buffering excess neurotransmitters at synapses through its mitochondria and metabolism. When astrocytic mitochondria are disrupted, neuronal hyperactivity may be triggered in AD. Also, since the $\beta$-oxidation process in astrocytic mitochondria exclusively consumes toxic fatty acids or lipid particles, astrocytic mitochondria play crucial roles in the removal of lipid particles associated with APOE in AD. The inflammatory status of microglia is determined by mitochondria and metabolic signaling in response to external stimuli. AD pathology cause metabolic reprogramming in microglia with the inflammatory response to become the activated or tolerance status.

\section{Astrocyte}

Astrocyte has crucial roles in the support of a neuron which includes the supply of metabolite, maintenance of synaptic plasticity and a control of neuronal activity in the brain (86). To preserve neural environment through buffering excessive glutamate as a neurotransmitter, it is known that astrocyte disposes of excessive released glutamate converting to glutamine by glutamine synthetase and the tricarboxylic acid (TCA) cycle of mitochondria (87). For this reason, it is seen that astrocytic mitochondria stay near glutamate transporter-1 (GLT-1, EAAT2) to regulate extracellular glutamate levels, which are followed by neuronal activation. When neuronal activity or glutamate uptake of astrocyte is inhibited, the proportion of mobile astrocytic mitochondria is increased instead of halting near GLT-1 to buffer glutamate $(88,89)$. In addition to mitochondria, glycolytic enzymes are co-localized with GLT-1. Although either the acute inhibition of glycolysis or the OXPHOS respiration in hippocampal slices cannot decrease glutamate uptake, simultaneous inhibition of both metabolisms reduce glutamate uptake, indicating that astrocytic metabolic state is a crucial factor for proper astrocytic functions (Fig. 2) (90). Using glia-specific mitochondrial gliotoxin being possible to impair selectively the OXPHOS system of glial cells, metabolic stress induced by mitochondrial dysfunction in glial cell inhibits the synaptic transmission (91). Thus, the differential metabolism of astrocyte satisfies the energetic demands of astrocytic functions, suggesting that the astrocytic metabolism has spatial and functional relation to the regulation of neuronal activity.

Astrocyte represents highly glycolytic metabolism compared to neurons $(92,93)$. For this reason, the pharmacological inhibition of glycolytic enzymes in astrocyte causes an accumulation of $A \beta$ near or within astrocytes in the brain (94). It suggests that the glycolytic metabolism of astrocyte contributes to progress of $\mathrm{AD}$ pathogenesis. It is reported that $20 \%$ of energy supplied to the brain comes from fatty acid oxidation, which is known to occur mainly in astrocyte (95). Hyperactive neurons release toxic fatty acids through lipoprotein-like particles with ApoE. At that point, the astrocytic mitochondria are used exclusively for $\beta$-oxidation consuming lipid droplets or free fatty acids as an energy source than for TCA cycle (71). These evidences suggest that toxic fatty acids released from hyperactive neurons by $A \beta$ can induce cytotoxicity, especially if they are not consumed due to 
damaged mitochondria of astrocyte. Moreover, if the secretion efficiency of toxic fatty acids depends on the ApoE polymorphism, it can be explained brain toxicity and high incidence of LOAD according to ApoE4 allele, which is a major risk factor for LOAD (Fig. 2).

Recently, research on the distinction of astrocytes between healthy individuals and $\mathrm{AD}$ has been investigated using an iPSC-derived model. Human iPSC-derived astrocyte model from early-onset familial AD (FAD) with PSEN1 M146L mutations or late-onset sporadic $\mathrm{AD}$ (SAD) with $\mathrm{ApoE4^{+/+ }}$ exhibits morphological differences, as compared to those from healthy individuals. Moreover, most induced astrocytes from AD patients appear fibroblast-like cell morphology and display astrocytic atrophy, suggesting the alterations of astrocyte contribute to the pathogenesis of AD (96). Studies on the dysfunction of astrocytic mitochondria in $A D$ have not investigated much more than those of other cell types in the brain. Astrocytes with PSEN1 $\triangle E 9$ mutations derived from AD patients made using an iPSC-derived model represent metabolic reprogramming from glycolysis to OXPHOS respiration, thereby increasing ROS production and reducing lactate secretion which supports neuronal functions (97). The astrocyte transcriptome comparing healthy control and AD subjects, which is isolated from the posterior cingulate region by laser capture microdissection following the staining with anti-Aldehyde dehydrogenase 1 family, member L1 (ALDH1L1) antibody specific to astrocyte cell type, describes that differentially expressed genes in astrocyte of $A D$ include mitochondria-related genes and immune responsive genes, indicating that astrocytic mitochondria are affected by the pathogenesis of AD (98).

In the AD brain, astrocytes have been reported to be exposed to oxidative stress, resulting in DNA damage and functional disability $(99,100)$. The increase of oxidative stress in astrocytes can be detected in old hAPP model mice, suggesting that astrocytic dysfunction by increased oxidative stress can contribute to the progress of AD pathogenesis (101). Additionally, an exposure of $A \beta$ to astrocyte can induce mitochondrial fragmentation and depolarization, therefore leading to increased ROS production and metabolic impairment $(102,103)$. In addition, $A \beta$ decreases the mitochondrial membrane potential of astrocytes but not the neurons, indicating the vulnerability of astrocytic mitochondria in $A D$ (104). Another way of toxicity in astrocyte is an accumulation of poly-ADP-ribose polymers produced by poly-ADP-ribose polymerase that are activated by $A \beta$-induced oxidative stress. The increased poly-ADP-ribose polymers that limit the availability of nicotinamide adenine dinucleotide as substrate, are also known to reduce mitochondrial membrane potential and result in neuronal death (103).

\section{Microglia}

Microglia, brain-resident immune cell, respond to surrounding stimuli and alert the immune response. Furthermore, mitochondria are required for the inflammatory responses of microglia and determining their metabolic status (105). A short exposure of $A \beta$ to microglia induces acute inflammatory response, including production of cytokines and phagocytosis of $A \beta$. Microglia acutely treated with $A \beta$ undergo metabolic reprogramming from OXPHOS to glycolysis via mTOR-HIF- $1 \alpha$ pathway. In the $A D$ brain, a long-term exposure of $A \beta$ and senile plaques leads microglia to convert to a tolerance status, in which they have defective metabolic system and their inflammatory responses are reduced, indicating that health metabolic system is important to maintain inflammatory responses to external stimuli (Fig. 2) (106).

Using a method to generate iPSC-derived human microglia-like cells (iMGLs), the contribution of genetic backgrounds of AD, ApoE4, PSEN1 $\triangle E 9$, and APPswe, to functions and metabolism of iMGLs is elucidated. Both FAD mutations, PSEN1 $\triangle E 9$, and APPSwe, have no effect on metabolic reprogramming. However, ApoE4 iMGLs exhibit lower oxygen consumption rate and can result in a decrease in all mitochondrial parameters related to cellular respiration. In addition, ApoE4 iMGLs, but not PSEN1 $\triangle E 9$ or APPswe iMGLs show reduced phagocytic capability (107). Additionally, hypomorphic variants of TREM2, a rare risk factor for LOAD associated with microglial responses, regulate microglial metabolism via mTOR signaling. Microglia in TREM2-deficient 5XFAD model mice have been shown to exhibit an accumulation of autophagosomes and impaired mTOR signaling due to down-regulated energy metabolism. These results suggest that TREM2 and mTOR-mediated metabolic activation mediates the function of microglia, such as the removal of amyloid plaques (Fig. 2) (108).

The mitochondria homeostasis is important to determine microglial inflammatory status, and its disruption can trigger neuronal death in neurodegenerative diseases. Recently, it has been suggested that microglial mitochondria are dysfunctional in neurodegenerative diseases, which are highly fragmented and released from microglia, thereby consequently inducing neuronal death. Dysfunctional mitochondria are detected in microglia-conditioned media when microglia are activated by $A \beta$. The treatment of P110 which is a selective inhibitor of mitochondrial fission and fragmentation, ameliorates glial activation and inflammatory responses in the brain of $A D$ model mice (Fig. 2) (109). Reduced signaling of mitophagy that eliminate dysfunctional mitochondria has been identified as one of the pathological features of AD. An accumulation of defective mitochondria in microglia increases the release of cytokines and inhibits the removal of amyloid plaques, promoting the inflammatory responses in the brain. The restoration of mitophagy can mitigate inflammation and reduce the activation of NLRP3-inflammasome. Qualitative control of mitochondrial in microglia can alleviate AD pathogenesis by inducing an appropriate inflammatory response in the brain (110). 


\section{CONCLUSION}

Mitochondrial dysfunction has been observed in the early stages of $A D$ before the onset of clinical symptoms and interferes with the metabolism of the brain. Both $A \beta$ and tau lesions induce the damage in various aspects of mitochondria, including the capacity of energy production, the control of homeostasis, and the transport of mitochondria along microtubules. Since various cell types that constitute the brain contribute to $\mathrm{AD}$ pathogenesis in different ways, an understanding of mitochondrial dysfunction in $A D$ needs to be interpreted based on cell type-specific functions. Mitochondria affected by $A \beta$ and tau pathologies cause a vicious cycle that induces the pathological features of $\mathrm{AD}$ pathogenesis at each cellular level. For this reason, a proper understanding of cell type-specific mitochondrial dysfunction contributing to $A D$ pathogenesis leads to elucidating the underlying mechanisms of $\mathrm{AD}$ pathogenesis and the discovery of therapeutic targets for AD.

\section{ACKNOWLEDGEMENTS}

This work was supported by the National Research Foundation of Korea (NRF) Grant funded by the Korean Government (MISP) (NRF-2018R1A2A1A19019062).

\section{CONFLICTS OF INTEREST}

The authors have no conflicting interests.

\section{REFERENCES}

1. Querfurth HW and LaFerla FM (2010) Alzheimer's disease. N Engl J Med 362, 329-344

2. Cao J, Hou J, Ping J and Cai D (2018) Advances in developing novel therapeutic strategies for Alzheimer's disease. Mol Neurodegener 13, 64

3. Sanabria-Castro A, Alvarado-Echeverria I and Monge-Bonilla C (2017) Molecular Pathogenesis of Alzheimer's Disease: An Update. Ann Neurosci 24, 46-54

4. Marcus C, Mena E and Subramaniam RM (2014) Brain PET in the diagnosis of Alzheimer's disease. Clin Nucl Med 39, e413-422; quiz e423-416

5. Mosconi L, Berti V, Glodzik L, Pupi A, De Santi S and de Leon MJ (2010) Pre-clinical detection of Alzheimer's disease using FDG-PET, with or without amyloid imaging. J Alzheimers Dis 20, 843-854

6. Patel JR and Brewer GJ (2003) Age-related changes in neuronal glucose uptake in response to glutamate and beta-amyloid. J Neurosci Res 72, 527-536

7. Beal MF (1995) Aging, energy, and oxidative stress in neurodegenerative diseases. Ann Neurol 38, 357-366

8. Mancuso M, Calsolaro V, Orsucci D et al (2009) Mitochondria, cognitive impairment, and Alzheimer's disease. Int J Alzheimers Dis 2009, 951548

9. Zhu X, Perry G, Smith MA and Wang X (2013) Abnormal mitochondrial dynamics in the pathogenesis of Alzheimer's disease. J Alzheimers Dis 33 Suppl 1, S253-262

10. Wang $X$, Su B, Zheng L, Perry G, Smith MA and Zhu X (2009) The role of abnormal mitochondrial dynamics in the pathogenesis of Alzheimer's disease. J Neurochem 109 Suppl 1, 153-159

11. Swerdlow RH (2018) Mitochondria and Mitochondrial Cascades in Alzheimer's Disease. J Alzheimers Dis 62, 1403-1416

12. Swerdlow RH, Burns JM and Khan SM (2010) The Alzheimer's disease mitochondrial cascade hypothesis. J Alzheimers Dis 20 Suppl 2, S265-279

13. Swerdlow RH, Burns JM and Khan SM (2014) The Alzheimer's disease mitochondrial cascade hypothesis: progress and perspectives. Biochim Biophys Acta 1842, 1219-1231

14. Cheng Y and Bai F (2018) The Association of Tau With Mitochondrial Dysfunction in Alzheimer's Disease. Front Neurosci 12, 163

15. Reddy PH and Beal MF (2008) Amyloid beta, mitochondrial dysfunction and synaptic damage: implications for cognitive decline in aging and Alzheimer's disease. Trends Mol Med 14, 45-53

16. Szablewski L (2017) Glucose Transporters in Brain: In Health and in Alzheimer's Disease. J Alzheimers Dis 55, 1307-1320

17. Sun J, Feng $X$, Liang D, Duan $Y$ and Lei $H$ (2012) Down-regulation of energy metabolism in Alzheimer's disease is a protective response of neurons to the microenvironment. J Alzheimers Dis 28, 389-402

18. Sonntag KC, Ryu WI, Amirault KM et al (2017) Late-onset Alzheimer's disease is associated with inherent changes in bioenergetics profiles. Sci Rep 7, 14038

19. Carvalho C, Cardoso S, Correia SC et al (2012) Metabolic alterations induced by sucrose intake and Alzheimer's disease promote similar brain mitochondrial abnormalities. Diabetes 61, 1234-1242

20. Zhang C, Rissman RA and Feng J (2015) Characterization of ATP alternations in an Alzheimer's disease transgenic mouse model. J Alzheimers Dis 44, 375-378

21. Cha MY, Han SH, Son SM et al (2012) Mitochondriaspecific accumulation of amyloid beta induces mitochondrial dysfunction leading to apoptotic cell death. PLoS One 7, e34929

22. Keeney JT, Ibrahimi S and Zhao L (2015) Human ApoE Isoforms Differentially Modulate Glucose and Amyloid Metabolic Pathways in Female Brain: Evidence of the Mechanism of Neuroprotection by ApoE2 and Implications for Alzheimer's Disease Prevention and Early Intervention. J Alzheimers Dis 48, 411-424

23. Wu L, Zhang $X$ and Zhao L (2018) Human ApoE Isoforms Differentially Modulate Brain Glucose and Ketone Body Metabolism: Implications for Alzheimer's Disease Risk Reduction and Early Intervention. J Neurosci 38, 6665-6681

24. Rhein V, Baysang G, Rao S et al (2009) Amyloid-beta leads to impaired cellular respiration, energy production and mitochondrial electron chain complex activities in human neuroblastoma cells. Cell Mol Neurobiol 29, 1063-1071 
25. Beck SJ, Guo L, Phensy A et al (2016) Deregulation of mitochondrial F1FO-ATP synthase via OSCP in Alzheimer's disease. Nat Commun 7, 11483

26. David DC, Hauptmann S, Scherping I et al (2005) Proteomic and functional analyses reveal a mitochondrial dysfunction in P301L tau transgenic mice. J Biol Chem 280, 23802-23814

27. Schulz KL, Eckert A, Rhein $V$ et al (2012) A new link to mitochondrial impairment in tauopathies. Mol Neurobiol 46, 205-216

28. Rhein V, Song X, Wiesner A et al (2009) Amyloid-beta and tau synergistically impair the oxidative phosphorylation system in triple transgenic Alzheimer's disease mice. Proc Natl Acad Sci U S A 106, 20057-20062

29. Manczak M, Anekonda TS, Henson E, Park BS, Quinn J and Reddy PH (2006) Mitochondria are a direct site of A beta accumulation in Alzheimer's disease neurons: implications for free radical generation and oxidative damage in disease progression. Hum Mol Genet 15, 1437-1449

30. Caspersen C, Wang N, Yao J et al (2005) Mitochondrial Abeta: a potential focal point for neuronal metabolic dysfunction in Alzheimer's disease. FASEB J 19, 2040-2041

31. Devi L, Prabhu BM, Galati DF, Avadhani NG and Anandatheerthavarada HK (2006) Accumulation of amyloid precursor protein in the mitochondrial import channels of human Alzheimer's disease brain is associated with mitochondrial dysfunction. J Neurosci 26, 9057-9068

32. Hansson Petersen CA, Alikhani N, Behbahani $\mathrm{H}$ et al (2008) The amyloid beta-peptide is imported into mitochondria via the TOM import machinery and localized to mitochondrial cristae. Proc Natl Acad Sci U S A 105, 13145-13150

33. Chen JX and Yan SS (2010) Role of mitochondrial amyloid-beta in Alzheimer's disease. J Alzheimers Dis 20 Suppl 2, S569-578

34. Han SH, Park JC and Mook-Jung I (2016) Amyloid beta-interacting partners in Alzheimer's disease: From accomplices to possible therapeutic targets. Prog Neurobiol 137, 17-38

35. Yoshida M, Muneyuki E and Hisabori T (2001) ATP synthase-a marvellous rotary engine of the cell. Nat Rev Mol Cell Biol 2, 669-677

36. Cha MY, Cho HJ, Kim C et al (2015) Mitochondrial ATP synthase activity is impaired by suppressed OGlcNAcylation in Alzheimer's disease. Hum Mol Genet 24, 6492-6504

37. Halestrap A (2005) Biochemistry: a pore way to die. Nature 434, 578-579

38. Nicotra A and Parvez S (2002) Apoptotic molecules and MPTP-induced cell death. Neurotoxicol Teratol 24, 599-605

39. Zamzami N, Larochette $\mathrm{N}$ and Kroemer G (2005) Mitochondrial permeability transition in apoptosis and necrosis. Cell Death Differ 12 Suppl 2, 1478-1480

40. Javadov S and Kuznetsov A (2013) Mitochondrial permeability transition and cell death: the role of cyclophilin d. Front Physiol 4, 76
41. Du H, Guo L, Fang F et al (2008) Cyclophilin D deficiency attenuates mitochondrial and neuronal perturbation and ameliorates learning and memory in Alzheimer's disease. Nat Med 14, 1097-1105

42. Lustbader JW, Cirilli M, Lin C et al (2004) ABAD directly links Abeta to mitochondrial toxicity in Alzheimer's disease. Science 304, 448-452

43. Vogtle FN, Wortelkamp S, Zahedi RP et al (2009) Global analysis of the mitochondrial $\mathrm{N}$-proteome identifies a processing peptidase critical for protein stability. Cell $139,428-439$

44. Mossmann D, Vogtle FN, Taskin AA et al (2014) Amyloid-beta peptide induces mitochondrial dysfunction by inhibition of preprotein maturation. Cell Metab 20, 662-669

45. Mishra P and Chan DC (2016) Metabolic regulation of mitochondrial dynamics. J Cell Biol 212, 379-387

46. Youle RJ and van der Bliek AM (2012) Mitochondrial fission, fusion, and stress. Science 337, 1062-1065

47. Zhang L, Trushin S, Christensen TA et al (2016) Altered brain energetics induces mitochondrial fission arrest in Alzheimer's Disease. Sci Rep 6, 18725

48. Shah SI, Paine JG, Perez C and Ullah G (2019) Mitochondrial fragmentation and network architecture in degenerative diseases. PLoS One 14, e0223014

49. Wang X, Su B, Lee HG et al (2009) Impaired balance of mitochondrial fission and fusion in Alzheimer's disease. J Neurosci 29, 9090-9103

50. Tyumentsev MA, Stefanova NA, Kiseleva EV and Kolosova NG (2018) Mitochondria with Morphology Characteristic for Alzheimer's Disease Patients Are Found in the Brain of OXYS Rats. Biochemistry (Mosc) 83, 1083-1088

51. Trushina E (2016) A shape shifting organelle: unusual mitochondrial phenotype determined with threedimensional electron microscopy reconstruction. Neural Regen Res 11, 900-901

52. Xie H, Guan J, Borrelli LA, Xu J, Serrano-Pozo A and Bacskai BJ (2013) Mitochondrial alterations near amyloid plaques in an Alzheimer's disease mouse model. J Neurosci 33, 17042-17051

53. Perez MJ, Ponce DP, Osorio-Fuentealba C, Behrens MI and Quintanilla RA (2017) Mitochondrial Bioenergetics Is Altered in Fibroblasts from Patients with Sporadic Alzheimer's Disease. Front Neurosci 11, 553

54. Manczak M, Calkins MJ and Reddy PH (2011) Impaired mitochondrial dynamics and abnormal interaction of amyloid beta with mitochondrial protein Drp1 in neurons from patients with Alzheimer's disease: implications for neuronal damage. Hum Mol Genet 20, 2495-2509

55. Joshi AU, Saw NL, Shamloo M and Mochly-Rosen D (2018) Drp1/Fis1 interaction mediates mitochondrial dysfunction, bioenergetic failure and cognitive decline in Alzheimer's disease. Oncotarget 9, 6128-6143

56. Baek SH, Park SJ, Jeong JI et al (2017) Inhibition of Drp1 Ameliorates Synaptic Depression, Abeta Deposition, and Cognitive Impairment in an Alzheimer's Disease Model. J Neurosci 37, 5099-5110

57. Wang X, Su B, Siedlak SL et al (2008) Amyloid-beta 
overproduction causes abnormal mitochondrial dynamics via differential modulation of mitochondrial fission/fusion proteins. Proc Natl Acad Sci U S A 105, 19318-19323

58. Knott $A B$, Perkins $G$, Schwarzenbacher R and Bossy-Wetzel E (2008) Mitochondrial fragmentation in neurodegeneration. Nat Rev Neurosci 9, 505-518

59. Westermann B (2009) Nitric oxide links mitochondrial fission to Alzheimer's disease. Sci Signal 2, pe29

60. Cho DH, Nakamura T, Fang J et al (2009) S-nitrosylation of Drp1 mediates beta-amyloid-related mitochondrial fission and neuronal injury. Science 324, 102-105

61. Kang S, Byun J, Son SM and Mook-Jung I (2018) Thrombospondin-1 protects against Abeta-induced mitochondrial fragmentation and dysfunction in hippocampal cells. Cell Death Discov 4, 31

62. Manczak M and Reddy PH (2012) Abnormal interaction between the mitochondrial fission protein Drp1 and hyperphosphorylated tau in Alzheimer's disease neurons: implications for mitochondrial dysfunction and neuronal damage. Hum Mol Genet 21, 2538-2547

63. Kandimalla R, Manczak M, Fry D, Suneetha Y, Sesaki $H$ and Reddy PH (2016) Reduced dynamin-related protein 1 protects against phosphorylated Tau-induced mitochondrial dysfunction and synaptic damage in Alzheimer's disease. Hum Mol Genet 25, 4881-4897

64. Perez MJ, Vergara-Pulgar K, Jara C, Cabezas-Opazo F and Quintanilla RA (2018) Caspase-Cleaved Tau Impairs Mitochondrial Dynamics in Alzheimer's Disease. Mol Neurobiol 55, 1004-1018

65. Byun J, Son SM, Cha MY et al (2015) CR6-interacting factor 1 is a key regulator in Abeta-induced mitochondrial disruption and pathogenesis of Alzheimer's disease. Cell Death Differ 22, 959-973

66. Kim C, Choi H, Jung ES et al (2012) HDAC6 inhibitor blocks amyloid beta-induced impairment of mitochondrial transport in hippocampal neurons. PLoS One 7, e42983

67. Shahpasand K, Uemura I, Saito T et al (2012) Regulation of mitochondrial transport and inter-microtubule spacing by tau phosphorylation at the sites hyperphosphorylated in Alzheimer's disease. J Neurosci 32, 2430-2441

68. Kopeikina KJ, Carlson GA, Pitstick R et al (2011) Tau accumulation causes mitochondrial distribution deficits in neurons in a mouse model of tauopathy and in human Alzheimer's disease brain. Am J Pathol 179, 2071-2082

69. Stamer K, Vogel R, Thies E, Mandelkow E and Mandelkow EM (2002) Tau blocks traffic of organelles, neurofilaments, and APP vesicles in neurons and enhances oxidative stress. J Cell Biol 156, 1051-1063

70. Fecher C, Trovo L, Muller SA et al (2019) Cell-typespecific profiling of brain mitochondria reveals functional and molecular diversity. Nat Neurosci 22, 1731-1742

71. Ioannou MS, Jackson J, Sheu SH et al (2019) Neuron-Astrocyte Metabolic Coupling Protects against Activity-Induced Fatty Acid Toxicity. Cell 177, 15221535 e1514

72. Park J, Choi H, Min JS et al (2013) Mitochondrial dynamics modulate the expression of pro-inflammatory mediators in microglial cells. J Neurochem 127, 221-232
73. Orihuela R, McPherson CA and Harry GJ (2016) Microglial M1/M2 polarization and metabolic states. $\mathrm{Br} J$ Pharmacol 173, 649-665

74. Vos M, Lauwers E and Verstreken P (2010) Synaptic mitochondria in synaptic transmission and organization of vesicle pools in health and disease. Front Synaptic Neurosci 2, 139

75. Davey GP, Peuchen S and Clark JB (1998) Energy thresholds in brain mitochondria. Potential involvement in neurodegeneration. J Biol Chem 273, 12753-12757

76. Brown MR, Sullivan PG and Geddes JW (2006) Synaptic mitochondria are more susceptible to $\mathrm{Ca} 2+$ overload than nonsynaptic mitochondria. J Biol Chem 281, 11658-11668

77. Zott B, Simon MM, Hong W et al (2019) A vicious cycle of beta amyloid-dependent neuronal hyperactivation. Science 365, 559-565

78. Du H, Guo L, Yan S, Sosunov AA, McKhann GM and Yan SS (2010) Early deficits in synaptic mitochondria in an Alzheimer's disease mouse model. Proc Natl Acad Sci U S A 107, 18670-18675

79. Pickett EK, Rose J, McCrory C et al (2018) Regionspecific depletion of synaptic mitochondria in the brains of patients with Alzheimer's disease. Acta Neuropathol $136,747-757$

80. Jadiya P, Kolmetzky DW, Tomar D et al (2019) Impaired mitochondrial calcium efflux contributes to disease progression in models of Alzheimer's disease. Nat Commun 10, 3885

81. Lee SH, Kim KR, Ryu SY et al (2012) Impaired short-term plasticity in mossy fiber synapses caused by mitochondrial dysfunction of dentate granule cells is the earliest synaptic deficit in a mouse model of Alzheimer's disease. J Neurosci 32, 5953-5963

82. Lee SH, Lutz D, Mossalam M, Bolshakov VY, Frotscher $M$ and Shen J (2017) Presenilins regulate synaptic plasticity and mitochondrial calcium homeostasis in the hippocampal mossy fiber pathway. Mol Neurodegener 12,48

83. Gazit N, Vertkin I, Shapira I et al (2016) IGF-1 Receptor Differentially Regulates Spontaneous and Evoked Transmission via Mitochondria at Hippocampal Synapses. Neuron 89, 583-597

84. Moloney AM, Griffin RJ, Timmons S, O'Connor R, Ravid $\mathrm{R}$ and O'Neill C (2010) Defects in IGF-1 receptor, insulin receptor and IRS-1/2 in Alzheimer's disease indicate possible resistance to IGF-1 and insulin signalling. Neurobiol Aging 31, 224-243

85. Zhang B, Tang XC and Zhang HY (2013) Alternations of central insulin-like growth factor-1 sensitivity in APP/PS1 transgenic mice and neuronal models. J Neurosci Res $91,717-725$

86. Allen NJ and Eroglu C (2017) Cell Biology of Astrocyte-Synapse Interactions. Neuron 96, 697-708

87. Rose CF, Verkhratsky A and Parpura V (2013) Astrocyte glutamine synthetase: pivotal in health and disease. Biochem Soc Trans 41, 1518-1524

88. Jackson JG, O'Donnell JC, Takano H, Coulter DA and Robinson MB (2014) Neuronal activity and glutamate uptake decrease mitochondrial mobility in astrocytes 
and position mitochondria near glutamate transporters. J Neurosci 34, 1613-1624

89. Xu NJ, Bao L, Fan HP et al (2003) Morphine withdrawal increases glutamate uptake and surface expression of glutamate transporter GLT1 at hippocampal synapses. J Neurosci 23, 4775-4784

90. Genda EN, Jackson JG, Sheldon AL et al (2011) Co-compartmentalization of the astroglial glutamate transporter, GLT-1, with glycolytic enzymes and mitochondria. J Neurosci 31, 18275-18288

91. Canals S, Larrosa B, Pintor J, Mena MA and Herreras O (2008) Metabolic challenge to glia activates an adenosine-mediated safety mechanism that promotes neuronal survival by delaying the onset of spreading depression waves. J Cereb Blood Flow Metab 28, 1835-1844

92. Belanger M, Allaman I and Magistretti PJ (2011) Brain energy metabolism: focus on astrocyte-neuron metabolic cooperation. Cell Metab 14, 724-738

93. Bouzier-Sore AK and Pellerin L (2013) Unraveling the complex metabolic nature of astrocytes. Front Cell Neurosci 7, 179

94. Fu W, Shi D, Westaway D and Jhamandas JH (2015) Bioenergetic mechanisms in astrocytes may contribute to amyloid plaque deposition and toxicity. J Biol Chem 290, 12504-12513

95. Ebert D, Haller RG and Walton ME (2003) Energy contribution of octanoate to intact rat brain metabolism measured by $13 \mathrm{C}$ nuclear magnetic resonance spectroscopy. J Neurosci 23, 5928-5935

96. Jones VC, Atkinson-Dell R, Verkhratsky A and Mohamet L (2017) Aberrant iPSC-derived human astrocytes in Alzheimer's disease. Cell Death Dis 8, e2696

97. Oksanen M, Petersen AJ, Naumenko N et al (2017) PSEN1 Mutant iPSC-Derived Model Reveals Severe Astrocyte Pathology in Alzheimer's Disease. Stem Cell Reports 9, 1885-1897

98. Sekar S, McDonald J, Cuyugan L et al (2015) Alzheimer's disease is associated with altered expression of genes involved in immune response and mitochondrial processes in astrocytes. Neurobiol Aging 36, 583-591

99. Myung NH, Zhu X, Kruman, Il et al (2008) Evidence of DNA damage in Alzheimer disease: phosphorylation of histone H2AX in astrocytes. Age (Dordr) 30, 209-215
100. Simpson JE, Ince PG, Haynes LJ et al (2010) Population variation in oxidative stress and astrocyte DNA damage in relation to Alzheimer-type pathology in the ageing brain. Neuropathol Appl Neurobiol 36, 25-40

101. Lee HP, Pancholi N, Esposito L et al (2012) Early induction of oxidative stress in mouse model of Alzheimer disease with reduced mitochondrial superoxide dismutase activity. PLoS One 7, e28033

102. Sarkar P, Zaja I, Bienengraeber M et al (2014) Epoxyeicosatrienoic acids pretreatment improves amyloid beta-induced mitochondrial dysfunction in cultured rat hippocampal astrocytes. Am J Physiol Heart Circ Physiol 306, $\mathrm{H} 475-484$

103. Abeti R, Abramov AY and Duchen MR (2011) Beta-amyloid activates PARP causing astrocytic metabolic failure and neuronal death. Brain 134, 1658-1672

104. Abramov AY, Canevari L and Duchen MR (2004) Beta-amyloid peptides induce mitochondrial dysfunction and oxidative stress in astrocytes and death of neurons through activation of NADPH oxidase. J Neurosci 24, 565-575

105. Culmsee C, Michels S, Scheu S, Arolt V, Dannlowski U and Alferink J (2018) Mitochondria, Microglia, and the Immune System-How Are They Linked in Affective Disorders? Front Psychiatry 9, 739

106. Baik SH, Kang S, Lee W et al (2019) A Breakdown in Metabolic Reprogramming Causes Microglia Dysfunction in Alzheimer's Disease. Cell Metab 30, 493-507 e496

107. Konttinen $\mathrm{H}$, Cabral-da-Silva MEC, Ohtonen $\mathrm{S}$ et al (2019) PSEN1DeltaE9, APPswe, and APOE4 Confer Disparate Phenotypes in Human iPSC-Derived Microglia. Stem Cell Reports 13, 669-683

108. Ulland TK, Song WM, Huang SC et al (2017) TREM2 Maintains Microglial Metabolic Fitness in Alzheimer's Disease. Cell 170, 649-663 e613

109. Joshi AU, Minhas PS, Liddelow SA et al (2019) Fragmented mitochondria released from microglia trigger A1 astrocytic response and propagate inflammatory neurodegeneration. Nat Neurosci 22, 1635-1648

110. Fang EF, Hou Y, Palikaras K et al (2019) Mitophagy inhibits amyloid-beta and tau pathology and reverses cognitive deficits in models of Alzheimer's disease. Nat Neurosci 22, 401-412 